\title{
Quantitative information in decay curves obtained with a pulsed ion mobility spectrometer
}

\author{
Wolfgang Baether, ${ }^{a}$ Stefan Zimmermann ${ }^{b}$ and Frank Gunzer ${ }^{* c}$ \\ Received 16th December 2011, Accepted 27th March 2012 \\ DOI: 10.1039/c2an16260a
}

Ion mobility spectrometry (IMS) is well known for its very high sensitivity, and thus IMS spectra are commonly used in the identification of trace gases. Extracting quantitative information from IMS spectra is, in contrast, difficult, especially regarding the reproducibility due to the nature of the processes involved in the measurement of the spectra. Here we present data extracted from signal decay curves obtained with a pulsed IMS, which can support the determination of substance concentrations in the lower ppb range with good stability.

\section{Introduction}

Ion mobility spectrometry (IMS) is a well-known method for detecting hazardous compounds in air. Typical applications include the detection of chemical warfare agents, explosives, and drugs of abuse. Detection limits in the low part per billion range, fast response times, and simple instrumentation have made this technique quite popular, especially in the field of portable detectors. ${ }^{1}$ Standard commercial devices use a radioactive electron source to ionize the sample molecules, which are then accelerated into a drift tube, where they collide with ambient air molecules and thus reach a constant drift velocity. This velocity depends on a number of parameters, which include the mass and the collision cross-section of the molecule. The consequence is that in general different substances reach a different velocity, and so they need a characteristic flight time to cross the drift tube. IMS devices are limited in their resolving power which reaches typically values between 40 and 60 , but their advantage is the high sensitivity achieved with their quite simple setup.

The ionization of the sample molecules is an indirect ionization, as the free electrons of the (radioactive) source in a first step create the so-called reactant ions, which then ionize the sample molecules via charge transfer reactions. The reactant ions are formed via a cascade of reactions involving especially the nitrogen and water molecules present in ambient air. The result is positively charged water-clusters and negatively charged $\mathrm{O}_{2}{ }^{-}$-clusters. Both form peaks in IMS spectra which are called reactant ion peaks (RIP). The IMS is operated in either a positive mode, where only the positively charged ions are accelerated

${ }^{a}$ Research Unit, Drägerwerk $A G$ \& Co. KGaA, Moislinger Allee 53-55, 23542 Lübeck, Germany

${ }^{b}$ Institute of Electrical Engineering and Measurement Technology, Leibniz University Hannover, Appelstr. 9a, 30167 Hannover, Germany

'Physics Department, German University in Cairo, Entrance El Tagamoa El Khames, New Cairo City, Cairo, Egypt. E-mail: frank.gunzer@guc. edu.eg; Fax: +2027581 041; Tel: +2027589 9908 through the drift tube towards the detector, or in a negative mode, where only the negatively charged ions are detected. ${ }^{2}$ Due to the charge transfer reaction between sample molecules and RIP molecules and the general conservation of charges, it is in principle possible to form a relation between the ratio of signal intensities (RIP and analyte) and analyte concentration. Examples are the detection of TNT, ${ }^{3}$ acetone, ${ }^{4}$ and ethanol, ${ }^{5}$ but also more complex substances such as morphine and noscapine. ${ }^{6}$ An interesting application is the cleaning validation and verification of manufacturing equipment in the pharmaceutical industry. ${ }^{7,8}$

In general, using the charge balance and thus the ratios of signal intensities for quantitative interpretations becomes difficult due to the number of reactions involved and their related uncertainties. As the underlying mechanisms are so complex, artificial neural networks (ANN) are a typical, more advanced approach to obtain calibration curves for quantitative IMS measurements since their application does not require the full understanding of the signal forming principles. ${ }^{9,10}$ The ANN are trained with a number of IMS spectra of known analyte concentrations. But using just signal intensities in order to determine analyte concentrations shows typically problems regarding the reproducibility, and variation of up to $25 \%$ has been reported in the literature. Advanced statistical approaches like principal component regression and partial least squares seem to offer improvement for this problem. ${ }^{11}$

The IMS spectra used in these different methods are obtained in a continuous mode with electron sources that continuously produce free electrons. In our setup we use an electron gun that produces short electron pulses, so that the ion signal's decay can be observed after the electron beam and thus the ionizing process has stopped. The decay characteristic depends on the substance itself, but also on other parameters, one being the analyte concentration. In such a manner we can determine concentration dependent parameters which are not only based on absolute signal intensity relations. In this paper we describe how the decay curves behave with respect to concentration changes for the 
substances ethyl methyl ketone, ethanol, dimethyl methylphosphonate (DMMP) and 2,4-toluenediisocyanate (TDI). After a short presentation of the setup, decay curves of these substances will demonstrate to what extent quantitative information can be extracted with good reliability from the decay behavior; the goal is to evaluate the decay characteristic as an orthogonal parameter that can help to increase the precision of such quantitative information obtained from IMS spectra.

\section{Experimental}

The setup has been intensively described in previous publications $^{12-14}$ so we give here only a brief summary. We used a drift tube and an amplifier from a modified Draeger Ion Mobility Spectrometer GSM with a $3 \mathrm{~mm}$ long reaction region, a $5.5 \mathrm{~cm}$ long drift region and a $0.5 \mathrm{~mm}$ long collector region. The device is operated in ambient air with corresponding temperature and pressure. At a frequency of $30 \mathrm{~Hz}$, a voltage pulse was used to inject the ions after the ionization process (caused by free electrons provided by an electron gun) from the reaction region (in which a small field of $6 \mathrm{~V} \mathrm{~cm}^{-1}$ was present to prevent drift losses before injection) into the drift region, where a permanent field of about $200 \mathrm{~V} \mathrm{~cm}^{-1}$ forced the ions towards the collector region. On their way through the drift region the ions collided with the air molecules (the drift tube was operated at atmospheric pressure) with an air flow of $300 \mathrm{ml} \mathrm{min}^{-1}$ that flowed in the opposite direction of the ions' motion. The flow rate was controlled by a mass flow controller (MFC). In the collector region the ions experienced a field of $1000 \mathrm{~V} \mathrm{~cm}^{-1}$ that moved them towards the detector, which was formed by a simple Faraday cup. The resolving power of the device was $c a$. 40, so that the different peaks were well separated from each other (e.g. ethanol: RIP $2.07 \mathrm{~cm}^{2} \mathrm{~V}^{-1} \mathrm{~s}^{-1}$, monomer $1.93 \mathrm{~cm}^{2} \mathrm{~V}^{-1} \mathrm{~s}^{-1}$, dimer $1.71 \mathrm{~cm}^{2} \mathrm{~V}^{-1} \mathrm{~s}^{-1}$, and peak width FWHM $0.04 \mathrm{~cm}^{2} \mathrm{~V}^{-1} \mathrm{~s}^{-1}$ ).

The free electrons forming the RIP ions were emitted by the electron gun in short pulses of $10 \mu$ s length with a frequency of $30 \mathrm{~Hz}$. The potential difference between the gun and the reaction region was $8 \mathrm{kV}$ so that the average kinetic energy of the electrons was close to that of $\beta$-radiation emitted by commercial tritium sources. The electron beam left the source through a source punch with a diameter of about $10 \mathrm{~mm}$.

The different concentrations of the analyte were produced by mixing dry air (water concentration at $292 \mathrm{~K}$ less than $50 \mathrm{ppm}$, i.e. less than $0.3 \%$ relative humidity) with a mixture composed of dry air and analyte (produced by mixing analyte gas evaporating in a permeation oven into a constant air flow of $100 \mathrm{ml} \mathrm{min}^{-1}$ ). The concentrations have been verified with different laboratory typical procedures.

\section{Results and discussion}

A general observation is that the decay curve, i.e. the dependence of the signal intensity on the delay time introduced in-between ion production (when the electron beam stops) and ion extraction, changes with the analyte concentration. With lower concentration the maximum intensity normally becomes lower. At relatively high concentrations the decay curve decays exponentially, but at certain low concentrations the intensity first increases with increasing delay time, and then decreases exponentially after reaching the maximum intensity. In this state, lowering the concentrations shifts the decay time necessary to reach maximum intensity towards longer delay times, and the increase towards the maximum intensity as well as the decay afterwards behave more slowly. ${ }^{13-17}$ Both are a result of the changed behavior of ion production via RIP ions and ion number reduction via recombination reactions, the consequence being that the whole decay curve becomes wider and shifts towards longer decay times. ${ }^{16}$ Fig. 1 shows this behavior for ethyl methyl ketone. This substance is measured in the positive mode of the IMS (ionization via water clusters), and in this mode it is quite common to observe monomer- and proton bound dimer-signals of the analyte. The behavior of the decay curves is thus more complicated due to the relation between the monomer and dimer signals, but the general trend described before is obvious. The intensities shown in this figure are the maximum peak intensities of the analyte peak in the corresponding IMS spectrum, with each IMS spectrum being the average of 16 measured IMS spectra.

As the absolute intensity is not of interest here, but the decay behavior itself, the decay curves (which typically look also for the other substances like those in Fig. 1) have been normalized so that the maximum of each curve reached $100 \%$. Three distinct effects, which are caused by concentration reduction and that can already be seen in Fig. 1 for example, become then clearer.

The maximum intensity (i.e. 100\% in the normalized form) is much stronger than the initial intensity at delay time $t=0 \mathrm{~ms}$ (e.g. in Fig. 1, dimer signal: $100 \%$, ca. $360 \mathrm{mV}$, at $t=0 \mathrm{~ms}$ for $165 \mathrm{ppb}$ since the maximum is already reached at a delay time $t=0 \mathrm{~ms}$, and for $15 \mathrm{ppb} c a .10 \%$ at $t=0 \mathrm{~ms}$, i.e. $7 \mathrm{mV}$ compared to a maximum of $c a .70 \mathrm{mV}$ at $t=2 \mathrm{~ms}$ ), the location of the maximum intensity shifts to longer delay times, and the decay after the maximum becomes slower so that the curves of different concentrations are clearly separated from each other.

Thus it is possible to choose certain points on the normalized decay curve that can represent the analyte concentration. Since the decay curves are quite stable with respect to external influences, ${ }^{13,16}$ these points correspondingly are quite stable, too.

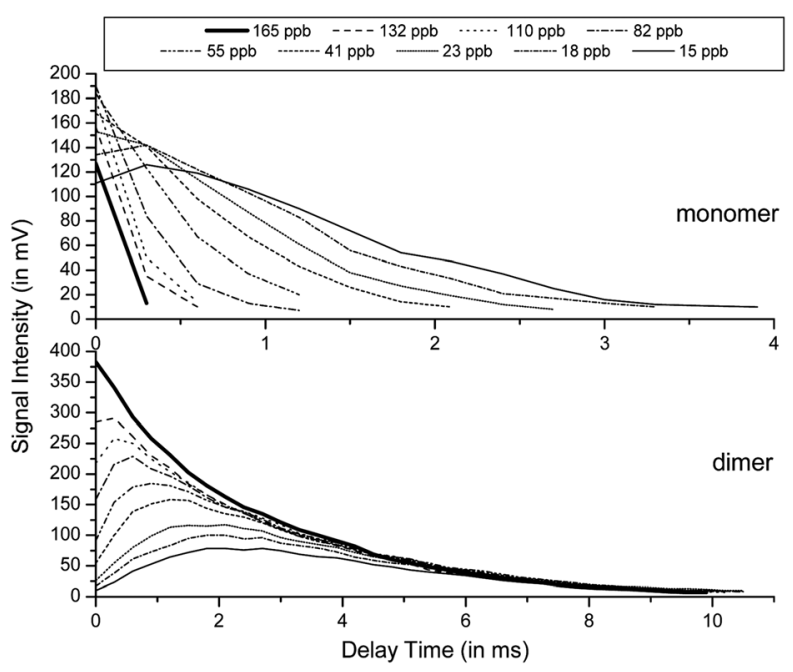

Fig. 1 Signal decay depending on the concentration, shown for ethyl methyl ketone. 
For ethyl methyl ketone we have chosen one point with fixed delay time (as described before the intensity at $t=0 \mathrm{~ms}$, called $I(0)$ ) and one point where the intensity was constant (delay time at which the intensity was $50 \%$ of the maximum intensity, called $t_{50 \%}$ ) on the normalized decay curve in order to demonstrate the corresponding dependence on concentration. Fig. 2 shows the dependence of these values for the dimer signal. Since the decay curves also depend on the strength of an electric field in the reaction region, ${ }^{14}$ the figure shows additionally the behavior for two different field strengths.

As can be seen, the $t_{50 \%}$ values become smaller with increasing concentration, the curve descends in a monotonic fashion. Increasing the field strength shifts the decay curve to faster times so that a different curve is obtained, but with a similarly monotonic behavior. The error bars show the variation typically observed in repeated measurements. At lower concentrations the curves show a steeper slope than at higher concentrations. Thus the method is limited towards high concentrations: when the slope becomes too small the curve no longer allows to discriminate safely between different concentrations. Towards lower concentrations the error increases and thus limits the applicability. But over the range investigated here ( $c a .20 \mathrm{ppb}$ to $160 \mathrm{ppb}$ ) the parameter $t_{50 \%}$ allows determination of the concentration with a precision of at least $5 \mathrm{ppb}$.

The second parameter, $I(0)$, increases with increasing concentration, as outlined before; it does not show any dependence on the electric field strength in this case. The slope is also monotonic, but changes in a stronger fashion at $c a .140 \mathrm{ppb}$. This is the point where the maximum intensity is already reached at a delay time of $0 \mathrm{~ms}$, and while the absolute value still changes (see Fig. 1 again), the value extracted from the normalized curve remains at $100 \%$; thus the limit for using this parameter is reached. Towards lower concentrations the error increases and limits thus this parameter, but again a precision of at least $5 \mathrm{ppb}$ is possible over the whole range.

Fig. 3 shows now the same parameters for the monomer signal. The monomer decays much faster than the dimer, and the

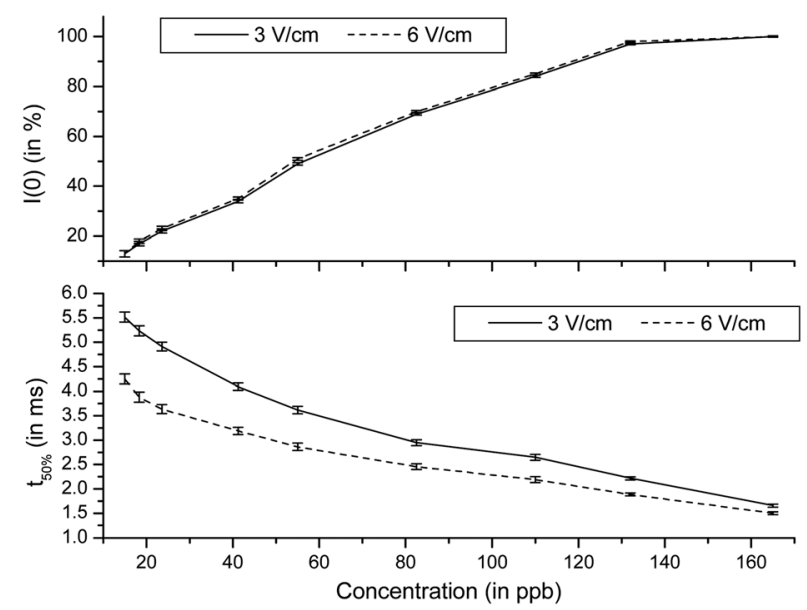

Fig. 2 Dependence of the intensity at $t=0 \mathrm{~ms}$ delay time $I(0)$ and the delay time necessary to reduce the intensity to $50 \%, t_{50 \%}$, on the concentration for two different electric field strengths in the reaction region, shown for the ethyl methyl ketone dimer (extracted from normalized signal intensities curves).

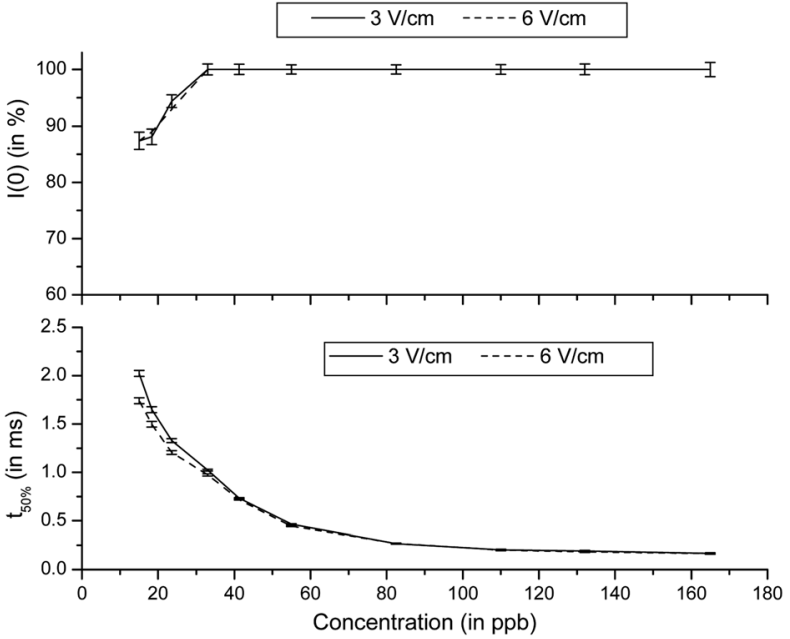

Fig. 3 Dependence of $I(0)$ and $t_{50 \%}$ on the concentration, shown for the monomer signal of ethyl methyl ketone.

normalized curve does not vary very much with the concentration. Only below $c a$. $80 \mathrm{ppb}$ the curve of $t_{50 \%}$ shows a slope large enough so that this parameter allows for the determination of the analyte concentration. The maximum intensity is for most concentrations already reached at $t=0 \mathrm{~ms}$ delay (see also Fig. 1, monomer graph, showing this with absolute intensities), and thus $I(0)$ is for most concentrations not a useful parameter either. Only below $40 \mathrm{ppb}$ the maximum intensity shifts to longer, nonzero delay times with decreasing concentration and can thus be used to determine the latter one in normalized decay curves.

Ethyl methyl ketone shows in the monomer and dimer signals the limitations of using the normalized decay curve to determine the analyte concentration. The method has a limit towards higher concentrations: when the IMS signal is strong (i.e. the concentration is high, since the signal intensity in general decreases with lower concentration), it will mainly change its intensity with concentration, and not in the behavior of the normalized decay curve (the decay is so fast that the variation of the decay itself with concentration is low, correspondingly the normalized curve shows only small variations). In general it can be said, that when the concentration is so low that the maximum (dimer) intensity is reached at a delay time which is not $0 \mathrm{~ms}$ anymore, then the maximum intensity has been reached (as an example see Fig. 1, 165 and $132 \mathrm{ppb}$ ). From this concentration on towards lower concentrations, at least $I(0)$ forms a suitable parameter. The other parameter $t_{50 \%}$ might still be useful at slightly higher concentrations, but the slope of the decay curve is then normally changing too little with the concentration so that the precision of $t_{50 \%}$ is very much reduced.

Since the limits of the method depend on the IMS signal strength, it is clear that different substances will show different limits. Ethanol shows only for the monomer signal a suitable behavior, albeit with different concentration limits compared to ethyl methyl ketone. Fig. 4 shows the corresponding graphs. Here the range is $c a$. $5 \mathrm{ppb}$ to $65 \mathrm{ppb}$ for $t_{50 \%}$, for $I(0)$ it is $c a .5$ ppb to $25 \mathrm{ppb}$, both with a precision of better than $5 \mathrm{ppb}$, especially below $20 \mathrm{ppb}$ since the magnitude of the slope for each curve is quite large. Both curves do not change significantly with 


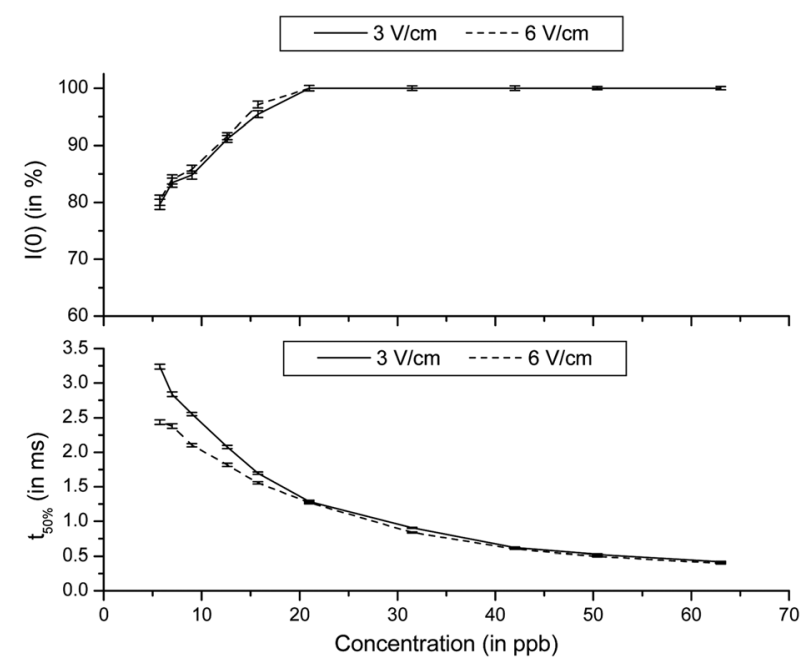

Fig. 4 Dependence of $I(0)$ and $t_{50 \%}$ on the concentration, shown for ethanol, monomer signal.

the field strength so that it is not possible to obtain a different curve as in the case of ethyl methyl ketone. The reason why ethanol has these lower limits is that the signal strength of ethanol is quite strong even with relatively low concentrations, i.e. the number of ions produced is quite high in comparison.

Another example investigated by our group is DMMP. DMMP shows strong signal decay variations with the concentration, but again only the monomer signal shows a suitable behavior that can be used to determine the analyte concentration. Furthermore, $I(0)$ is not a suitable parameter anymore. But since the whole decay curve can in principle be used, we chose the intensity at a delay time of $1.2 \mathrm{~ms}, I(1.2 \mathrm{~ms})$, instead of $I(0)$ (Fig. 5).

Only for a field strength of $3 \mathrm{~V} \mathrm{~cm}^{-1}$ the curves show a good monotonic decay. Since $I(1.2 \mathrm{~ms})$ is the signal intensity after reaching the maximum intensity, and since the maximum intensity is reached at shorter delay times with increasing concentration, $I(1.2 \mathrm{~ms})$ has a negative slope. The precision is here again $5 \mathrm{ppb}$ for a range between $10 \mathrm{ppb}$ and $115 \mathrm{ppb}$, but problematic

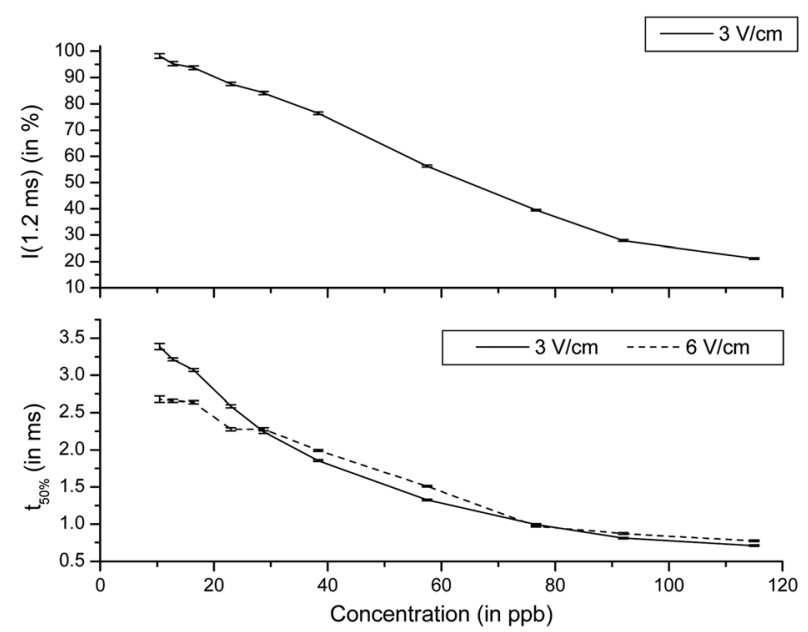

Fig. 5 Dependence of $I(1.2 \mathrm{~ms})$ and $t_{50 \%}$ on the concentration, shown for DMMP, monomer signal. at concentrations below $20 \mathrm{ppb}$. The other parameter $t_{50 \%}$ does not show a problem here, due to the steep curve it allows actually for a much better precision.

The last substance discussed in this paper is TDI. TDI is in contrast to the other substances not measured in the positive mode of the IMS device, but in the negative. In this mode there are no dimer signals, the signal formation is thus less complex. TDI shows only a moderate dependence of the decay curves on the concentrations, but still it allows us to find good parameters for this interpretation. This time it is not $t_{50 \%}$ but $t_{80 \%}$, i.e. the time at which the concentration reached $80 \%$ of its maximum value, and $I(3.0 \mathrm{~ms})$ (Fig. 6). Only with these parameters was it possible to obtain curves that allow a precision of better than $5 \mathrm{ppb}$. The range for this substance is from $5 \mathrm{ppb}$ to $80 \mathrm{ppb}$, and especially below $10 \mathrm{ppb}$ the slope is so steep that the precision is theoretically much better than $5 \mathrm{ppb}$. TDI thus shows that also in the negative mode the concentration dependence of the decay curve is strong enough in order to use it to determine the concentration, even in absence of dimer forming processes like in the case of the other substances. Furthermore it shows that in principle the whole decay curve can be used to find good parameters for the purpose of quantitative information extraction from IMS spectra.

The described behavior has only been shown for single substances. Further investigation needs to show how much this behavior changes if other substances are present. It can be expected that the dependencies change since the quantities of the RIP ions which are involved in the recombination reactions will be different. But similar influences would also change the outcome when using the intensity ratio based approaches; ${ }^{3-11}$ in these publications the influence was also investigated for only one substance or compositions of the same substances. In this aspect the here proposed method does not mean an improvement but suffers from the same restrictions. The advantage is on the one hand having other, orthogonal parameters to determine the concentration, and on the other hand the stability of these parameters. It is thus not meant to replace the other purely intensity based approaches, but to support them especially for

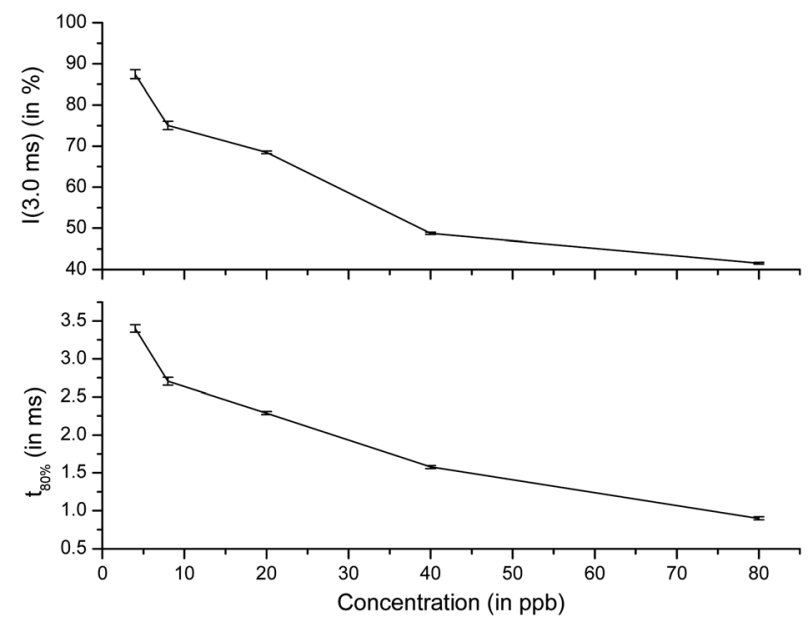

Fig. 6 Dependence of $I(3.0 \mathrm{~ms})$ and $t_{80 \%}$ on the concentration in the case of TDI (monomer, recorded in the negative IMS mode, field strength $\left.3 \mathrm{~V} \mathrm{~cm}^{-1}\right)$. 
the low concentrations shown here. A pulsed IMS allows us to determine both, intensity ratios (for this case measured without delay) and decay curves (measured by scanning over the delay times). The response time for the here proposed method is the same as that for standard IMS (for the measurement of $t_{50 \%}$ and $t_{80 \%}$ the maximum delay time was $6 \mathrm{~ms}$, delay time scans were performed in steps of $300 \mu$ s so 20 steps were necessary to cover this range; the device was operated at $30 \mathrm{~Hz}$ with averaging over 16 spectra, leading to a maximum scan time of $c a$. 12 seconds; for the fixed points $I(0)$ and $I(1.2 \mathrm{~ms})$ where no scanning is necessary it is correspondingly about $600 \mu$ s due to the averaging).

\section{Conclusion}

The normalized decay curves behave quite stably with respect to external parameters, and also regarding run-to-run stability or long-time stability even if the absolute signal intensities are quite different. Their decay behavior depends also on the analyte concentration, and thus these curves can be used for quantitative analysis. In this paper we have shown that for ethyl methyl ketone, ethanol, DMMP and TDI the decay curves allow us to extract the analyte concentration from the shifts of the decay curve to longer decay times with lower concentration. Thus either points with a certain ratio with respect to $100 \%$ intensity, or points recorded at a fixed delay time can be used. The practical concentration range depends on the substance and the signal type (monomer, dimer); for ethyl methyl ketone it was the largest with a range from 20 to $165 \mathrm{ppb}$, for ethanol it was the smallest with a range of $5 \mathrm{ppb}$ to $65 \mathrm{ppb}$, both with a precision better than 5 ppb. Using absolute intensities, the range could possibly be increased towards higher concentrations, since the absolute values keep changing with the concentration even when it is high; but the decay is then so fast that its behavior varies only slightly with the concentration, with the consequence that normalized curves would show a quite similar decay; the focus of this paper on the other hand is concentrated on the decay behavior alone and thus only normalized intensity curves have been analyzed, leaving the absolute decay curves open for further research and possible improvement. Since both neural networks and advanced statistics can improve their performance if supported by good orthogonal parameters, decay characteristics measured with pulsed IMS devices are a good candidate to lead to further improvement regarding precision in the lower ppb range not only but also in these methods.

Possible applications are situations where a fast and sensitive gas analyzer is needed, and additionally portability is required, but where standard IMS due to their lack of providing quantitative information could not be used; such applications are common in e.g. environmental monitoring.

\section{Notes and references}

1 G. A. Eiceman and Z. Karpas, Ion Mobility Spectrometry, CRC Press, Boca Raton, USA, 2005.

2 H. Borsdorf, T. Mayer, M. Zarejousheghani and G. A. Eiceman, Appl. Spectrosc. Rev., 2011, 46, 472-521.

3 F. Garofolo, F. Marziali, V. Migliozzi and A. Stama, Rapid Commun. Mass Spectrom., 1996, 10, 1321-1326.

4 T. Khayamian, M. Tabrizchi and N. Taj, Fresenius' J. Anal. Chem., 2001, 370, 1114-1116.

5 H.-Y. Han, G.-D. Huang, S.-P. Jin, P.-C. Zheng and G.-H. Xu, J. Environ. Sci., 2007, 19, 751-755.

6 T. Khayamian, M. Tabrizchi and M. T. Jafari, Talanta, 2006, 69, 795799.

7 M. A. Strege, J. Kozerski, N. Juarbe and P. Mahoney, Anal. Chem., 2008, 80, 3040-3044.

8 M. A. Strege, Anal. Chem., 2009, 81, 4576-4580.

9 Z. Boger and Z. Karpas, J. Chem. Inf. Comput. Sci., 1994, 34, 576580.

10 P. Zheng, P. B. Harrington and D. M. Davis, Chemom. Intell. Lab. Syst., 1996, 33, 121-132.

11 C. G. Fraga, D. R. Kerr and D. A. Atkinson, Analyst, 2009, 134, 2329-2337.

12 F. Gunzer, A. Ulrich and W. Baether, Int. J. Ion Mobility Spectrom., 2010, 13, 9-16.

13 F. Gunzer, S. Zimmermann and W. Baether, Anal. Chem., 2010, 82, 3756-3763.

14 W. Baether, S. Zimmermann and F. Gunzer, Int. J. Ion Mobility Spectrom., 2010, 13, 95-101.

15 F. Gunzer, W. Baether and S. Zimmermann, Int. J. Ion Mobility Spectrom., 2011, 14, 99-107.

16 W. Baether, S. Zimmermann and F. Gunzer, IEEE Sens. J., 2011, DOI: 10.1109/JSEN.2011.2179117.

17 P. Cochems, F. Gunzer, J. Langejuergen, A. Heptner and S. Zimmermann, Int. J. Ion Mobility Spectrom., 2012, 15, 31-39. 